Pesq. Vet. Bras. 36(7):581-586, julho 2016 DOI: $10.1590 /$ S0100-736X2016000700003

\title{
Avaliação ectoparasitológica, hematológica e histopatológica de traíras Hoplias malabaricus Bloch, 1794 provenientes de açudes localizados no município de Sumé, estado da Paraíba ${ }^{1}$
}

\author{
Maria G.S. Bernardino², Edijanio G. Silva ${ }^{2}$, Tercio I.C. Bezerra ${ }^{3}$, Ricardo B. Lucena ${ }^{4}$ \\ e Fabiana Satake ${ }^{4}$
}

\begin{abstract}
Bernardino M.G.S., Silva E.G., Bezerra T.I.C., Lucena R.B. \& Satake F. 2016. [Ectoparasitologic, hematologic and histopathologic assessment of Hoplias malabaricus Bloch, 1794 from ponds located in Sumé municipality, state of Paraíba, Brazil.] Avaliação ectoparasitológica, hematológica e histopatológica de traíras Hoplias malabaricus Bloch, 1794 provenientes de açudes localizados no município de Sumé, estado da Paraíba. Pesquisa Veterinária Brasileira 36(7):581-586. Universidade Federal da Paraíba, Campus II, Centro de Ciências Agrárias, Cidade Universitária, Rodovia PB-079, Areia, PB 58397-000, Brazil. E-mail: maryangel_ufpb@hotmail.com

Hoplias malabaricus Bloch, 1794 is one of the fish species most commonly found in the micro-region ponds of Cariri in Paraíba; however there are no studies in the region on its parasitic fauna. Due to this, the study aimed to characterize the ectoparasitologic fauna, blood profile and histopathological findings of the gills of $H$. malabaricus from four ponds in Sumé/PB. At each pond 10 specimens were captured and limnological water analysis was performed as the biometrics of specimens and blood samples were collected by caudal venipuncture. Parasite collection methods were used according to literature recommendations. Also gill samples were collected for histopathological assessment. Comparison of the averages were made by Tukey test at a probability of 5\%. The results show that $H$. malabaricus ectoparasite fauna from ponds located in Sumé/PB is diverse, once various ectoparasites taxons are identified on body surface and gills. The parasitic fauna promoted hematological and histopathological changes in the gills. The results show also an epibiosis relationship of Epistylis sp. with Lernaea cyprinacea and branchiurans crustaceans on $H$. malabaricus.
\end{abstract}

INDEX TERMS: Wolf fish, Hoplias malabaricus, fishes, ectoparasites, epibiosis, Lernaea cyprinacea, branchiurans crustaceans.

RESUMO.- Hoplias malabaricus Bloch, 1794 é uma das espécies de peixes mais comumente encontrada nos açudes da Microrregião do Cariri Paraibano, todavia ainda não existem estudos na região sobre a sua fauna parasitária. Em virtude disso, o estudo objetivou caracterizar a fauna ecto-

\footnotetext{
${ }^{1}$ Recebido em 5 de julho de 2015.

Aceito para publicação em 6 de abril de 2016.

2 Mestrando em Ciência Animal, Universidade Federal da Paraíba (UFPB), Campus II, Centro de Ciências Agrárias, Cidade Universitária, Rodovia PB-079, Areia, PB 58397-000, Brasil. *Autor para correspondência: maryangel_ufpb@hotmail.com

${ }^{3}$ Mestre em Ciência Animal, UFPB, Campus II, Centro de Ciências Agrárias, Cidade Universitária, Areia, PB 58397-000, Brasil.

${ }^{4}$ Docente do Departamento de Ciências Veterinárias, UFPB, Areia, PB 58397-000, Brasil.
}

parasitológica, o perfil hematológico e os achados histopatológicos das brânquias de H. malabaricus provenientes de quatro açudes localizados no município de Sumé/PB. Em cada açude foram capturados 10 exemplares e foi realizada a análise limnológica da água. Realizou-se a biometria dos exemplares e foram colhidas amostras sanguíneas por venopunção caudal. Os métodos de coleta de parasitos foram realizados segundo as recomendações da literatura. Além disso, foram coletadas amostras de brânquias para avaliação histopatológica. A comparação das médias foi feita pelo Teste Tukey a $5 \%$ de probabilidade. Os resultados mostram que a ectoparasitofauna de $H$. malabaricus provenientes de açudes localizados no município de Sumé/PB apresentou-se diversificada, sendo identificados vários táxons de ectoparasitos na superfície corporal e brânquias. A fauna 
parasitária promoveu alterações hematológicas e histopatológicas nas brânquias. Além disso, os resultados evidenciam uma relação de epibiose de Epistylis sp. com Lernaea cyprinacea e crustáceos branquiúros em $H$. malabaricus.

TERMOS DE INDEXAÇÃO: Traíra, Hoplias malabaricus, peixes, ectoparasitos, epibiose, Lernaea cyprinacea, crustáceos branquiúros.

\section{INTRODUÇÃO}

Hoplias malabaricus Bloch, 1794 (Characiformes, Erythrynidae) conhecida popularmente como traíra, possui uma grande capacidade de adaptação a diferentes condições hidrológicas (Gonçalves \& Morelli 2000), sendo encontrada em toda a América do Sul (Barros et al. 2007). É uma das espécies de peixes mais comumente observada nos açudes da Microrregião do Cariri Paraibano, sendo largamente comercializada em feiras livres.

Nos últimos anos, nota-se um aumento no número de estudos sobre a fauna parasitária da traíra, sendo os parasitas monogenéticos (Malacarne \& Godoi 2012, Corrêa et al. 2013, Graça et al. 2013), digenéticos (Costa et al. 2015) e nematoides (Martins et al. 2005, Meneguetti et al. 2013) os mais relatados nessa espécie, todavia ainda não existem estudos sobre a fauna parasitária de traíras residentes em lagoas na Microrregião do Cariri Paraibano.

A gravidade das lesões promovidas pelos parasitos nos hospedeiros vai depender de vários fatores, como por exemplo, o grupo parasitário em questão, o modo como atua e o sítio de infestação (Pavanelli et al 2008). Os efeitos nos hospedeiros podem resultar em retardo no crescimento, alterações no quadro hematológico (Tavares-Dias et al. 2009) e predispor à infecções secundárias (Pavanelli et al 2008). Além disso, algumas espécies quando em altas infestações nas brânquias podem causar lesões graves que podem evoluir para insuficiência respiratória e morte do hospedeiro (Vasconcelos \& Tavares-Dias 2014).

Segundo Luque (2004), quando os peixes se encontram intensamente parasitados ou com lesões profundas, dificilmente recuperam sua normalidade após tratamentos. Por isso, uma forma eficiente de monitorar o estado fisiológico e imunológico dos peixes hospedeiros é verificar a presença de parasitos associado a avaliação dos parâmetros hematológicos. Além disso, o fato das brânquias serem um sítio importante de infestação parasitária e por reagirem fortemente à presença de parasitas (Pavanelli et al. 2008) é aconselhável investigar a presença de lesões histopatológicas neste órgão e bem como, avaliar o grau das lesões quando presentes.

Em virtude disso, o estudo objetivou caracterizar a fauna ectoparasitológica, o perfil hematológico e os achados histopatológicos das brânquias de Hoplias malabaricus provenientes de açudes localizados no município de Sumé, Paraíba.

\section{MATERIAL E MÉTODOS}

Este estudo foi aprovado pelo Comitê de ética de experimentação Animal da Universidade Federal da Paraíba e aprovado, estando registrado sob protocolo $\mathrm{n}$ 0 307/13.
Área em estudo e captura dos peixes. As coletas foram realizadas em quatro açudes localizados no município de Sumé, Microrregião do Cariri Paraibano, Semiárido Nordestino: Açude de Sumé ( $7^{\circ} 40^{\prime} 10^{\prime \prime} \mathrm{S}$ e $\left.36^{\circ} 54^{\prime} 44^{\prime \prime} \mathrm{W}\right)$, Açude Passagem Rasa (7³5' $4^{\prime \prime} \mathrm{S}$ e $36^{\circ} 53^{\prime} 20^{\prime \prime} \mathrm{W}$ ), Poço Escuro (7 $7^{\circ} 33^{\prime} 47^{\prime \prime} \mathrm{S}$ e $36^{\circ} 58^{\prime} 36^{\prime \prime} \mathrm{W}$ ) e Açudinho ( $7^{\circ} 34^{\prime} 22^{\prime \prime} \mathrm{S}$ e $36^{\circ} 50^{\prime}$ 53"W). Em cada açude foram capturados 10 exemplares de Hoplias malabaricus, com tarrafas, boiões e/ou anzol.

Análise hematológica. Os peixes foram puncionados na veia caudal com seringas descartáveis contendo EDTA a 5\%. Imediatamente após a coleta foi confeccionada extensão sanguínea e a diluição (1:200) em solução de formol citrato para contagem de eritrócitos em câmara de Newbauer. 0 volume globular (VG) foi determinado pelo método do microhematócrito (Jain 1993) e a concentração de hemoglobina segundo recomendação de Hrubec \& Smith (2000). Os índices hematimétricos de Wintrobe: volume globular médio (VGM) e concentração de hemoglobina globular média (CHGM) foram determinados a partir de equações matemáticas (Kerr 2003). As extensões sanguíneas destinadas à determinação da leucometria global e diferencial foram coradas com May-Grünwald-Giemsa e as contagens de leucócitos e trombócitos realizadas segundo Martins et al. (2004). As análises laboratoriais foram realizadas no Laboratório de Patologia Clínica do Hospital Veterinário do Departamento de Ciências Veterinárias, Universidade Federal da Paraíba.

Avaliação parasitológica. A raspagem da superfície corporal foi realizada imediatamente após a captura dos peixes, evitando assim perda do material através de manipulação. Os peixes foram analisados quanto à presença de ectoparasitos visíveis a olho nu durante a análise externa e lesões características de parasitismo. A eutanásia dos peixes foi realizada por secção da medula espinhal e em seguida realizada a raspagem das brânquias. Em nenhum momento os peixes foram sedados, uma vez que o uso dos anestésicos pode reduzir o número de ectoparasitas (Callahan \& Noga 2002, Jerônimo et al 2011). A pesquisa por parasitos foi realizada em raspados frescos e em amostras acondicionadas em formol a 5\%, ambas em objetiva de 10X. Os animais foram necropsiados e os órgãos foram inspecionados macroscopicamente para sinais de alterações. Os crustáceos coletados foram conservados em álcool $70 \%$ e posteriormente, cada parasito foi examinado individualmente em microscopia óptica (objetiva de 4x e 10x). A identificação dos parasitos foi realizada segundo Pavanelli et al. (2008), Eiras et al. (2010) e Pavanelli et al. (2013).

Análise biométrica. Realizou-se a biometria dos exemplares, avaliando-se comprimento e peso totais. A partir destes dados foi calculado o fator de condição de Fulton (Rocha et al. 2005).

Análise histopatológica. Amostras de brânquias de cada espécime foram coletadas e fixadas em formol a $10 \%$, processadas rotineiramente, embebidas em parafina, cortadas a $5 \mu \mathrm{m}$, coradas com hematoxilina-eosina e examinados sob microscopia de luz. Foi utilizado fotomicroscópio para documentar as lesões encontradas. As amostras foram processadas no Laboratório de Patologia Animal do Hospital Veterinário do Departamento de Ciências Veterinárias, Universidade Federal da Paraíba.

Análise limnológica. Concomitantemente as capturas dos exemplares foram realizadas coletas de água dos açudes para avaliação limnológica. As características físico-químicas da água foram avaliadas, sendo aferidos temperatura e concentração de oxigênio dissolvido com oxímetro digital, o pH com pHmetro e a transparência da água foi medida com o disco de Secchi.

Análise estatística dos resultados. A taxa de prevalência foi calculada segundo Bush et al. (1997). Os dados foram submetidos à análise de variância e a comparação das médias foi feita pelo Teste Tukey a 5\% de probabilidade através do programa estatístico SAS 9.2.2. 


\section{RESULTADOS}

Nesse estudo foram observados oito táxons de parasitos em Hoplias malabaricus (Quadro 1), sendo que os peixes do Açudinho apresentaram maior diversidade parasitária em um total de sete táxons, seguido dos peixes do Açude de Sumé (6 táxons), Poço Escuro (5 táxons) e Passagem Rasa (2 táxons). Na superfície corporal, foram observados oito táxons de parasitos, em que Lernaea cyprinacea, Trichodina spp. e Epystilis sp. foram observados com a maior taxa de prevalência nos peixes do Açude de Sumé, Poço Escuro e Açudinho. Nas brânquias foram observados sete táxons de parasitos, com a maior taxa de prevalência de copepoditos de L. cyprinacea, Dactylogyrus spp., Trichodina spp. e Ergasilidae gen. sp. nos peixes do Açude Passagem Rasa e Açudinho. Das 40 traíras analisadas, 30 (75\%) apresentaram-se infestadas por L. cyprinacea, sendo observadas as três fases de desenvolvimento do copépode: náuplio, com a prevalência de $13,33 \%$, copepodito $(100 \%)$ e parasitos adultos $(73,33 \%)$. Formas adultas de L. cyprinacea foram encontradas parasitando preferencialmente a região próxima às nadadeiras dos peixes, e raramente, fixas às brânquias. Todos os peixes infestados pelo copépode apresentaram lesões cutâneas hemorrágicas e inflamatórias decorrentes do ponto de fixação do parasito. Após a avaliação dos 70 parasitos adultos de L. cyprinacea, constatou-se que $51(72,86 \%)$ copépodes apresentaram Epistylis sp. fixados ao seu corpo $(p<0,05)$, evidenciando assim uma relação de epibiose. Esta relação também foi observada em crustáceos branquiúros (Fig.1).

Os resultados dos parâmetros hematológicos dos espécimes estudados estão expressos na Quadro 2. Após a avaliação dos parâmetros do eritrograma, constatou-se que os

Quadro 1. Taxa de prevalência (valores médios em \%) de parasitos encontrados na superfície do corpo e nas brânquias de espécimes de Hoplias malabaricus provenientes de açudes localizados no município de Sumé, estado da Paraíba

\begin{tabular}{|c|c|c|c|c|c|c|}
\hline \multirow[t]{2}{*}{ Local de infestação } & \multirow[t]{2}{*}{ Parasito } & \multicolumn{4}{|c|}{ Açudes } & \multirow[t]{2}{*}{ Média geral } \\
\hline & & Sumé & Passagem Rasa & Poço Escuro & Açudinho & \\
\hline \multirow[t]{8}{*}{ Superfície corporal } & Trichodina spp. & 80 & 40 & 70 & 90 & 70,0 \\
\hline & L. cyprinacea & 100 & 0 & 80 & 100 & 70,0 \\
\hline & Epystilis sp. & 50 & 0 & 70 & 90 & 52,5 \\
\hline & Argulus sp. & 50 & 0 & 0 & 20 & 17,5 \\
\hline & Dolops sp. & 60 & 0 & 0 & 0 & 15,0 \\
\hline & Lamproglena sp. & 20 & 0 & 0 & 10 & 7,5 \\
\hline & Ergasilidae gen. sp. & 0 & 0 & 10 & 20 & 7,5 \\
\hline & Dactylogyrus spp. & 0 & 0 & 10 & 10 & 5,0 \\
\hline \multirow[t]{7}{*}{ Brânquias } & Trichodina spp. & 20 & 0 & 50 & 100 & 42,5 \\
\hline & L.cyprinacea & 50 & 0 & 100 & 100 & 62,5 \\
\hline & Epistylis sp. & 10 & 0 & 10 & 50 & 17,5 \\
\hline & Argulus sp. & 10 & 0 & 0 & 0 & 2,5 \\
\hline & Lamproglena sp. & 10 & 0 & 0 & 10 & 5,0 \\
\hline & Ergasilidae gen. sp. & 0 & 0 & 90 & 40 & 32,5 \\
\hline & Dactylogyrus spp. & 0 & 40 & 100 & 90 & 57,5 \\
\hline Superfície corporal & Trichodina spp. & 90 & 40 & 70 & 90 & 72,5 \\
\hline \multirow[t]{7}{*}{ ou brânquias } & L. cyprinacea & 100 & 0 & 100 & 100 & 75,0 \\
\hline & Epistylis sp. & 60 & 0 & 70 & 90 & 55,0 \\
\hline & Argulus sp. & 60 & 0 & 0 & 20 & 20,0 \\
\hline & Dolops sp. & 60 & 0 & 0 & 0 & 15,0 \\
\hline & Lamproglena sp. & 20 & 0 & 0 & 20 & 10,0 \\
\hline & Ergasilidae gen. sp. & 0 & 0 & 90 & 40 & 32,5 \\
\hline & Dactylogyrus spp. & 0 & 40 & 100 & 90 & 57,5 \\
\hline
\end{tabular}

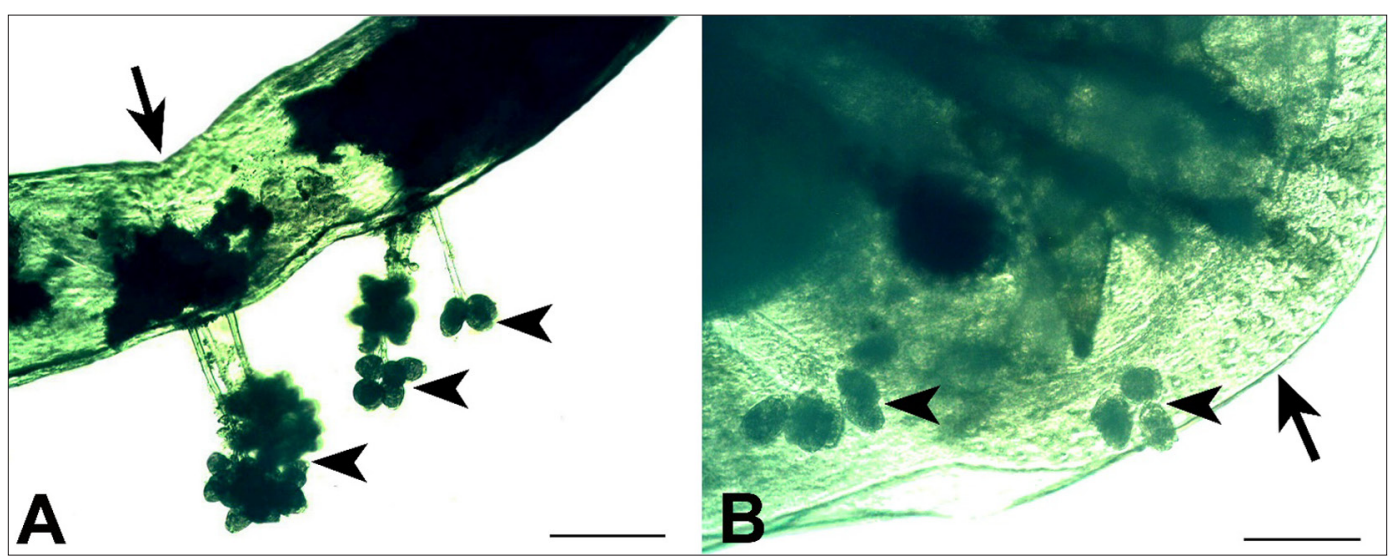

Fig.1. Fotomicrografias da relação de epibiose do Epystilis sp. com outros ectoparasitos. (A) Epystilis sp. (pontas de seta) fixados na superfície da Lernaea cyprinacea (seta). (B) Epystilis sp. (pontas de seta) fixados na superfície do Argulus sp. (seta). Barras: A) 600 4 m e B) $300 \mu \mathrm{m}$. 
peixes do açude Passagem Rasa apresentaram os maiores valores médios de volume globular, hematimetria e hemoglobinemia e os peixes do açude de Sumé apresentaram os menores valores médios $(p<0,05)$. Os peixes do Açude de Sumé apresentaram os maiores valores médios de monócitos e basófilos $(p<0,05)$. Evidenciou-se também um aumento significativo de células granulocíticas especiais nos

\section{Quadro 2. Valores médios dos resultados dos parâmetros} hematológicos de Hoplias malabaricus provenientes de açudes localizados no município de Sumé, Paraíba

\begin{tabular}{lccccc}
\hline & \multicolumn{4}{c}{ Açudes } & P \\
\cline { 2 - 5 } & $\begin{array}{c}\text { Sumé } \\
(\mathrm{n}=10)\end{array}$ & $\begin{array}{c}\text { Passagem } \\
\text { Rasa (n=10) }\end{array}$ & $\begin{array}{c}\text { Poço Escuro } \\
(\mathrm{n}=10)\end{array}$ & $\begin{array}{c}\text { Açudinho } \\
(\mathrm{n}=10)\end{array}$ \\
\hline VG (L/L) & $0,23^{\mathrm{b}}$ & $0,31^{\mathrm{a}}$ & $0,30^{\mathrm{ab}}$ & $0,28^{\mathrm{ab}}$ & 0,03 \\
Er (x10 $/ \mathrm{L})$ & $1,10^{\mathrm{b}}$ & $1,56^{\mathrm{a}}$ & $1,23^{\mathrm{ab}}$ & $1,11^{\mathrm{ab}}$ & $<0,01$ \\
Hb (g/L) & $40,8^{\mathrm{c}}$ & $70,2^{\mathrm{a}}$ & $61,9^{\mathrm{ab}}$ & $50,5^{\mathrm{bc}}$ & $<0,01$ \\
VGM (fL) & $224,62^{\mathrm{ab}}$ & $203,62^{\mathrm{b}}$ & $246,80^{\mathrm{ab}}$ & $256,79^{\mathrm{a}}$ & 0,01 \\
CHGM (\%) & $17,28^{\mathrm{b}}$ & $22,30^{\mathrm{a}}$ & $21,16^{\mathrm{a}}$ & $18,07^{\mathrm{b}}$ & $<0,01$ \\
PPT (g/L) & 57,8 & 53,6 & 49,0 & 48,4 & 0,27 \\
TROMB (x10 $/ \mathrm{L})$ & 36,08 & 37,52 & 47,71 & 48,43 & 0,15 \\
LG (x10 $/ \mathrm{L})$ & 54,58 & 32,40 & 46,39 & 41,51 & 0,18 \\
Monócito (x10 $/ \mathrm{L})$ & $5,89^{\mathrm{a}}$ & $3,08^{\mathrm{ab}}$ & $1,78^{\mathrm{b}}$ & $1,27^{\mathrm{b}}$ & $<0,01$ \\
Linfócito (x109/L) & 29,47 & 20,29 & 20,57 & 26,52 & 0,49 \\
Basófilo (x10 $/ \mathrm{L})$ & $0,16^{\mathrm{a}}$ & $0,01^{\mathrm{b}}$ & $0,02^{\mathrm{b}}$ & $0,02^{\mathrm{b}}$ & $<0,01$ \\
Eosinófilo (x10 $/ \mathrm{L})$ & 0,43 & 0,25 & 0,02 & 0,53 & 0,18 \\
Neutrófilo (x10 $/ \mathrm{L})$ & 18,18 & 8,68 & 10,67 & 10,96 & 0,09 \\
CGE (x10 $/ \mathrm{L})$ & $0,45^{\mathrm{ab}}$ & $0,08^{\mathrm{b}}$ & $1,45^{\mathrm{a}}$ & $0,40 \mathrm{a}^{\mathrm{b}}$ & 0,03
\end{tabular}

Onde: VG: volume globular, Er: Eritrócitos, Hb: hemoglobina, VGM: volume globular médio, CHGM: concentração de hemoglobina globular média, PPT: proteína plasmática total; TROMB: trombócitos. LG: leucometria global, CGE: célula granulocítica especial. Valores com letras diferentes na mesma linha diferem entre si pelo Teste Tukey a 5\% de significância. peixes dos Açudes de Sumé, Poço Escuro e Açudinho, em relação ao Açude Passagem Rasa $(p<0,05)$. Além disso, os maiores valores médios da leucometria global foram observados nos peixes do Açude de Sumé, Poço Escuro e Açudinho, em relação aos peixes do Açude Passagem Rasa, que não apresentaram alta infestação parasitária, todavia este aumento não foi significativo estatisticamente. A célula granulocítica especial foi o leucócito mais observado nas brânquias de traíras, seguido dos linfócitos (Fig.2).

Os peixes do Açudinho e Poço Escuro apresentaram os menores índices biométricos (Quadro 3) e as maiores taxas de prevalências de alterações histopatológicas nas brânquias (Quadro 4).

Os dados da análise individual da qualidade da água dos açudes estão expressos no Quadro 5.

Os resultados mostram que os peixes com maior diversidade e carga parasitária nas brânquias, provenientes do Açudinho e Poço Escuro, apresentaram concomitantemente os menores índices biométricos e maiores taxas de prevalências de alterações histopatológicas nas brânquias.

\section{DISCUSSÃo}

Este é o primeiro estudo epidemiológico relacionado à investigação da fauna parasitária em espécimes de Hoplias malabaricus na Paraíba. Nossos dados mostram que Lernaea cyprinacea está presente na região estudada, sendo encontrada em alta prevalência. Estes dados corroboram com a hipótese de que atualmente este parasito esteja disseminado em todos os cursos de água do Brasil (Eiras et al. 2010).

Quadro 3. Valores médios seguidos de desvio-padrão dos dados biométricos realizados em Hoplias malabaricus provenientes de açudes localizados no município de Sumé, Paraíba

\begin{tabular}{lcccc}
\hline & \multicolumn{3}{c}{ Açudes } \\
\cline { 2 - 5 } & Sumé & Passagem Rasa & Poço Escuro & Açudinho \\
\hline Peso (g) & $472,60^{\mathrm{a}} \pm 153,50$ & $366,60^{\mathrm{a}} \pm 77,08$ & $232,70^{\mathrm{b}} \pm 53,77$ & $164,30^{\mathrm{b}} \pm 57,30$ \\
Comprimento $(\mathrm{cm})$ & $34,40^{\mathrm{a}} \pm 2,65$ & $33,75^{\mathrm{a}} \pm 3,22$ & $30,75^{\mathrm{ab}} \pm 2,31$ & $28,00^{\mathrm{b}} \pm 4,08$ \\
FC de Fulton $\left(10^{2}\right)$ & $1,13^{\mathrm{a}} \pm 0,18$ & $0,95^{\mathrm{b}} \pm 0,10$ & $0,80^{\mathrm{bc}} \pm 0,14$ & $0,75^{\mathrm{c}} \pm 0,15$
\end{tabular}

Onde: FC - Fator de condição. Valores com letras diferentes na mesma linha diferem entre si pelo Teste Tukey a 5\% de significância.
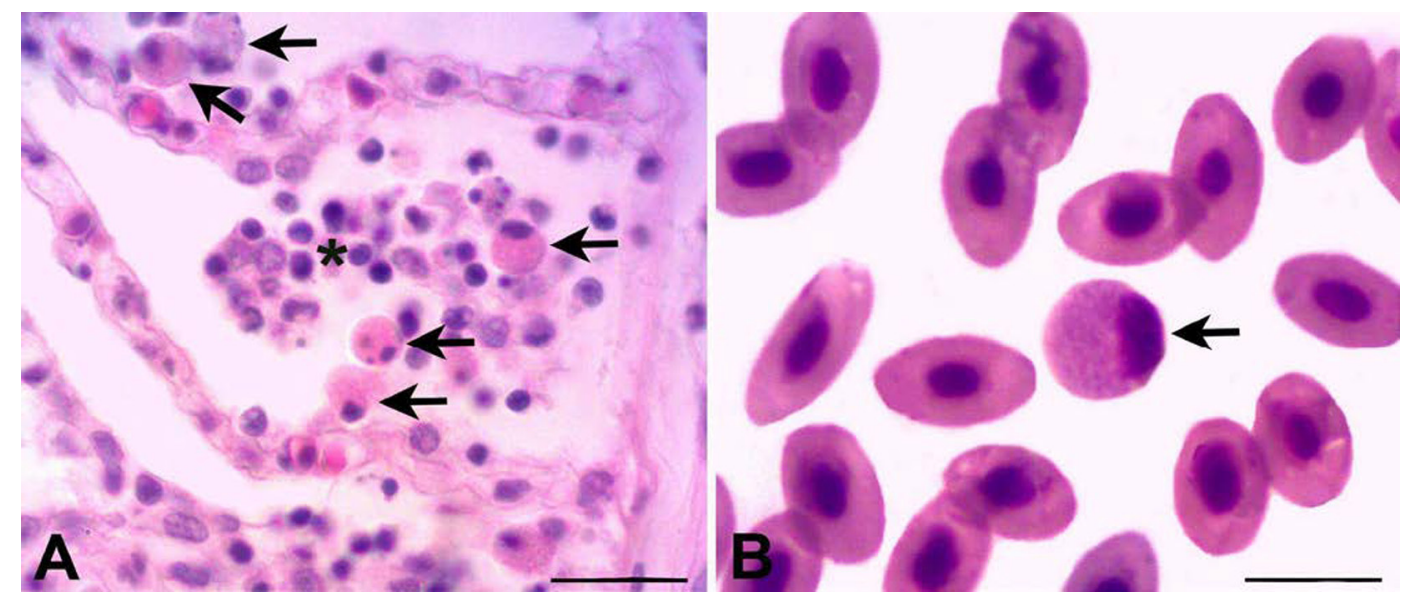

Fig.2. (A) Fotomicrografia da célula granulocítica especial (setas) e infiltrado linfocitário (asterisco) em brânquias de Hoplias malabaricus. (B) Fotomicrografia da célula granulocítica especial (seta) em esfregaço sanguíneo de Hoplias malabaricus. Coloração: A) Hematoxilina-eosina e B) May-Grünwald-Giemsa. Barras: A) $13 \mu \mathrm{m}$ e B) $10 \mu \mathrm{m}$. 
Quadro 4. Achados histopatológicos (resultados em \%) em brânquias de Hoplias malabaricus provenientes de açudes localizados no município de Sumé, Paraíba

\begin{tabular}{|c|c|c|c|c|c|}
\hline \multirow[t]{2}{*}{ Açude } & \multirow[t]{2}{*}{ Tipo da alteração } & \multirow[t]{2}{*}{$\%$} & \multicolumn{3}{|c|}{ Grau da alteração } \\
\hline & & & Leve & Moderado & Acentuado \\
\hline \multirow[t]{6}{*}{ Sumé } & Hiperplasia lamelar & 30 & 100 & 0 & 0 \\
\hline & Infiltrado inflamatório & 90 & 78 & 22 & 0 \\
\hline & Telangiectasia & 50 & 60 & 40 & 0 \\
\hline & Atrofia lamelar & 10 & 100 & 0 & 0 \\
\hline & Fusão lamelar & 10 & 100 & 0 & 0 \\
\hline & Média & 38 & 88 & 12 & 0 \\
\hline Passagem & Hiperplasia lamelar & 90 & 33 & 56 & 11 \\
\hline \multirow[t]{5}{*}{ Rasa } & Infiltrado inflamatório & 40 & 75 & 25 & 0 \\
\hline & Telangiectasia & 50 & 20 & 60 & 20 \\
\hline & Atrofia lamelar & 10 & 100 & 0 & 0 \\
\hline & Fusão lamelar & 10 & 100 & 0 & 0 \\
\hline & Média & 40 & 66 & 28 & 6 \\
\hline Poço & Hiperplasia lamelar & 100 & 20 & 70 & 10 \\
\hline \multirow[t]{5}{*}{ Escuro } & Infiltrado inflamatório & 70 & 29 & 57 & 14 \\
\hline & Telangiectasia & 30 & 67 & 33 & 0 \\
\hline & Atrofia lamelar & 60 & 17 & 83 & 0 \\
\hline & Fusão lamelar & 20 & 100 & 0 & 0 \\
\hline & Média & 56 & 47 & 48 & 5 \\
\hline \multirow[t]{6}{*}{ Açudinho } & Hiperplasia lamelar & 90 & 56 & 22 & 22 \\
\hline & Infiltrado inflamatório & 80 & 50 & 38 & 12 \\
\hline & Telangiectasia & 20 & 50 & 0 & 50 \\
\hline & Atrofia lamelar & 50 & 40 & 40 & 20 \\
\hline & Fusão lamelar & 30 & 67 & 0 & 33 \\
\hline & Média & 54 & 53 & 20 & 27 \\
\hline
\end{tabular}

Quadro 5. Valores médios dos parâmetros físico-químicos da água mensurados em açudes localizados no município de Sumé, Paraíba

\begin{tabular}{lcccc}
\hline Parâmetros & \multicolumn{4}{c}{ Açudes } \\
\cline { 2 - 5 } & Sumé & Passagem Rasa & Poço Escuro & Açudinho \\
\hline Temperatura $\left({ }^{\circ} \mathrm{C}\right)$ & 24,0 & 26,2 & 27,3 & 25,7 \\
pH & 8,55 & 8,35 & 8,5 & 8,4 \\
OD (mg/L) & 5,7 & 8,1 & 8,0 & 7,2 \\
Transparência (cm) & 130 & 11 & 28 & 34
\end{tabular}

Onde: OD = oxigênio dissolvido.

A fase de copepodito de L. cyprinacea que é considerada infestante (Eiras et al. 2010), foi observada em todos os peixes analisados mesmo quando os espécimes não apresentaram os parasitos adultos. Com isso, aconselha-se que seja realizada a pesquisa por parasitos de L. cyprinacea em microscopia óptica concomitante a análise externa nos peixes, pois a ausência da forma adulta não exclui o parasitismo por outras fases intermediárias.

Os náuplios são liberados após a eclosão dos ovos de $L$. cyprinacea na água, este estágio é considerado livre e não infestante (Kubitza \& Kubitza 2004). Isso explica a baixa prevalência observada, que provavelmente ocorreu por contaminação da água.

Os ectoparasitos observados em maior taxa de prevalência foram L. cyprinacea, Trichodina spp., Dactylogyrus spp., Epistylis spp. e Ergasilidae gen. sp. que possuem alto potencial patogênico (Pavanelli et al. 2008).

Outro fato relevante na investigação parasitária é a verificação das relações estabelecidas entre os parasitos, pois os resultados deste estudo mostram que há uma relação de epibiose do Epistylis sp. com a L. cyprinacea e com crustáceos branquiúros. Esse achado mostra que a presença ou ausência de uma determinada espécie de parasito pode influenciar na infestação por outras espécies. No entanto, são necessários estudos que esclareçam as consequências desta associação, uma vez que, tanto o protozoário, quanto o copépode pode promover lesões tegumentares hemorrágicas no hospedeiro (Gallio et al. 2007, Moyses et al. 2015).

A co-infestação por L. cyprinacea, Dolops sp. e Argulus sp. pode ter intensificado o quadro anêmico apresentado pelas traíras do açude de Sumé, uma vez que a ação desses parasitos pode promover hemorragias no local de fixação nos hospedeiros (Luque 2004, Pavanelli et al. 2008, Noor El-Deen et al. 2013).

Nos peixes com maior infestação parasitária foi observado um aumento significativo nos valores médios de células granulocíticas especiais na corrente circulatória, todavia, a função desse leucócito ainda é desconhecida. Alguns estudos relatam que este leucócito possui a habilidade de migrar para focos inflamatórios (Martins et al. 2009). Além disso, a célula granulocítica especial foi o leucócito mais comumente observado nas brânquias, evidenciando que este leucócito está ativamente envolvido nos mecanismos de defesa desses espécimes.

A função do basófilo ainda não está totalmente esclarecida nos peixes, mas há evidências de que esta célula possa realizar fagocitose, removendo debris celulares (Satake et al. 2009), assim como o monócito. 0 aumento desses leucócitos na corrente sanguínea dos peixes indica um processo inflamatório crônico. Essas alterações podem estar associadas as lesões inflamatórias promovidas por branquiúros e L. cyprinacea (Pavanelli et al 2008).

A lesão histopatológica mais observada foi a hiperplasia em lamelas secundárias, que geralmente está associada à presença de ectoparasitos. Entre estes, os mais implicados no desenvolvimento destas lesões são os monogenoides, Trichodina spp. (Zanolo \& Yamamura 2006, Luque 2004), Ergasilidae gen. sp. (Luque 2004) e os estágios intermediários de L. cyprinacea, no qual os copepoditos se movimentam ativamente na superfície do hospedeiro (Martins \& Souza Jr 1995).

As maiores taxas de prevalência de atrofia lamelar foram observadas em peixes infestados por Ergasilidae gen. sp. e se correlaciona com a patogenicidade do parasito nos hospedeiros. Uma vez que os ergasilídeos possuem um par de antenas modificadas que envolvem a porção final do filamento branquial, causando compressão do filamento, oclusão e rompimento de vasos sanguíneos (Kilian \& Avenant-Oldewage 2013).

A atividade parasitária nos hospedeiros apresentou-se com caráter debilitante, pois espécimes com as maiores taxas de infestação apresentaram os menores índices biométricos.

Os resultados da análise individual da água dos açudes quando comparados com os valores de referência do Conselho Nacional do Meio Ambiente - Conama (Resolução no 357 2005) mostram que o açude de Sumé, Passagem Rasa e Açudinho apresentaram temperatura baixa, média de $25,3^{\circ} \mathrm{C}$. Segundo Kubitza (2000), temperaturas abaixo de $27^{\circ} \mathrm{C}$ promovem redução do apetite e no crescimento, promovem depressão do sistema imunológico, predispondo ao ataque de organismos patogênicos. 


\section{CONCLUSÕES}

A partir do estudo realizado conclui-se que traíras (Hoplias malabaricus) provenientes de açudes localizados no município de Sumé, estado da Paraíba apresentaram uma ectoparasitofauna diversificada, sendo identificados vários táxons de ectoparasitos, tanto na superfície corporal, quanto nas brânquias.

A infestação parasitária promoveu alterações hematológicas e histopatológicas nas brânquias.

Os resultados evidenciam uma relação de epibiose de Epistylis sp. com L. cyprinacea e crustáceos branquiúros.

Agradecimentos.- Os autores agradecem ao Conselho Nacional de Desenvolvimento Científico e Tecnológico (CNPq) e à Coordenação de Aperfeiçoamento de Pessoal de Nível Superior (CAPES) pela concessão de bolsas. Ao Programa Nacional de Cooperação Acadêmica (Procad/CNPq) pelo auxílio financeiro para publicação (88881.068412/2014-01). Aos colaboradores Edilson Galdino da Silva, Santiago Benites de Pádua, George Luís Dias dos Santos e Minervina Regina da Silva que contribuíram para realização dessa pesquisa.

\section{REFERÊNCIAS}

Barros L.A., Moraes Filho J. \& Oliveira R.L. 2007. Larvas de nematóides de importância zoonótica encontradas em traíras (Hoplias malabaricus Bloch, 1794) no município de Santo Antonio do Leverger, MT. Arq. Bras. Med. Vet. Zootec. 59(2):533-535.

Bush A.O., Lafferty K.D., Lotz J.M. \& Shostak A.W. 1997. Parasitology meets ecology on its own terms (Revised by Margolis et al.). J. Parasitol. 83(4): 575-583.

Callahan H.A. \& Noga E.J. 2002. Tricaine dramatically reduces the ability to diagnose protozoan ectoparasite (Ichthyobodo necator) infections. J. Fish Dis. 25:433-437.

Corrêa L.L., Karling L.C., Takemoto R.M., Ceccarelli P.S. \& Ueta M.T. 2013. Hematological parameters of Hoplias malabaricus (Characiformes: Erythrinidae) parasitized by Monogenea in lagoons in Pirassununga, Brazil. Revta Bras. Parasitol. Vet. 22(4):457-462.

Costa D.P.C., Monteiro C.M. \& Brasil-Sato M.C. 2015. Digenea of Hoplias intermedius and Hoplias malabaricus (Actinopterygii, Erythrinidae) from upper São Francisco River, Brazil. Braz. J. Vet. Parasitol. 24(2):129-135.

Eiras J.C., Takemoto R.M. \& Pavanelli G.C. 2010. Diversidade de Parasitas de Água Doce do Brasil. Clichetec, Maringá. 333p.

Gallio M., Silva A.S. \& Monteiro S.G. 2007. Parasitismo por Lernaea cyprinacea em Astyanax bimaculatus provenientes de um açude no município de Antonio Prado, Rio Grande do Sul. Acta Scient. Vet. 35(2):209-212.

Gonçalves P.C. \& Morelli S. 2000. Estudo cariotípico da população de Hoplias malabaricus do Rio das Pedras. Disponível em <http://www.propp.ufu.br/pub/B>

Graça R.J., Ueda B.H., Oda F.H. \& Takemoto R.M. 2013. Monogenea (Platyhelminthes) parasites from the gills of Hoplias aff. malabaricus (Bloch, 1794) (Pisces: Erythrinidae) in the Upper Paraná River Floodplain, States of Paraná and Mato Grosso do Sul, Brazil. Check List 9(6):14841487.

Hrubec T.C. \& Smith S.A. 2000. Hematology of fish, p.1120-1125. In: Feldman B.F., Zinkl J.G. \& Jain N.C. (Eds), Schalm's Veterinary Hematology. 5th ed. Lippincott Williams and Wilkins, Baltimore.

Jain N.C. 1993. Essentials of Veterinary Hematology. Lea and Febiger, Pennsylvania. $417 \mathrm{p}$.

Jerônimo G.T., Martins M.L., Ishikawa M.M., Ventura A.S. \& Tavares-Dias M. 2011. Métodos para Coleta de Parasitos de Peixes. Embrapa Amapá, Macapá. Disponível em <https://www.infoteca.cnptia.embrapa.br/bitstream/doc/905949/1/AP2011MetodoscoletaCircular.pdf>

Kerr M.G. 2003. Exames Laboratoriais em Medicina Veterinária: bioquímica clínica e hematologia. 2ª ed. Roca, São Paulo. 436p.
Kilian E. \& Avenant-Oldewage A. 2013. Infestation and pathological alterations by Ergasilus sarsi (Copepoda) on the Tanganyika killifish from Africa. J. Aquatic Anim. Health 25:237-242.

Kubitza F. 2000. Tilápia: tecnologia e planejamento na produção comercial. F. Kubitza, Jundiaí. 289p.

Kubitza F. \& Kubitza L.M.M. 2004. Principais Parasitoses e Denças dos Peixes Cultivados. 4aㅡ ed. F. Kubitza, Jundiaí. 110p.

Luque J.L. 2004. Biologia, epidemiologia e controle de parasitos de peixes. Revta Bras. Parasitol. Vet. 13(1):161-165.

Malacarne P.L.C. \& Godoi M.M.M.M. 2012. Monogenéticos parasitos de brânquias de Hoplias malabaricus (Traíra) e saúde animal na Amazônia Ocidental. Revta Bras. Ciênc. Amazônia 1(1):109-113.

Martins M.L., Myiazaki D.M.Y., Tavares-Dias M., Fenerick Jr J., Onaka E.M., Bozo F., Fujimoto R.Y. \& Moraes F.R. 2009. Caracterization of the acute inflammatory response in the hybrid tambacu (Piaractus mesopotamicus male x Colossoma macropomum female) (Osteichthyes). Braz. J. Biology 69(2):631-637.

Martins M.L., Onaka E.M. \& Fenerick Jr J. 2005. Larval Contracaecum sp. (Nematoda: Anisakidae) in Hoplias malabaricus and Hoplerythrinus unitaeniatus (Osteichthyes: Erythrinidae) of economic importance in occidental marshlands of Maranhão, Brazil. Vet. Parasitol. 127(1):51-59.

Martins M.L., Pilarsky F., Onaka E.M., Nomura D.T., Fenerick J., Ribeiro K., Myiazaki D.M.Y., Castro M.P. \& Malheiros E.B. 2004. Hematologia e resposta inflamatória aguda em Oreochromis niloticus (Osteichthyes: Cichlidae) submetida aos estímulos único e consecutivo de estresse de captura. Bol. Inst. Pesca 30(1):71-80.

Martins M.L. \& Souza Jr F.L. 1995. Infestação experimental em girinos de Rana catesbeiana shaw por copepoditos de Lernaea cyprinacea Linnaeus (Copepoda, Lernaeidae). Revta Bras. Zool. 12(3):619-625.

Meneguetti D.U.O., Laray M.P.O. \& Camargo L.M.A. 2013. Primeiro relato de larvas de Eustrongylides sp. (Nematoda: Dioctophymatidae) em Hoplias malabaricus (Characiformes: Erythrinidae) no Estado de Rondônia, Amazônia Ocidental, Brasil. Revta Pan-Amaz. Saude 4(3):55-58.

Moyses C.R.S., Spadacci-Morena D.D., Xavier J.G., Antonucci A.M. \& Lallo M.A. 2015. Ectocommensal and ectoparasites in goldfish Carassius auratus (Linnaeus, 1758) in farmed in the State of São Paulo. Braz. J. Vet. Parasitol. 24(3):283-289.

Noor El-deen A.I.E., Hassan A.H.M. \& Mahmoud A.E. 2013. Studies on Lernaeosis Affecting Cultured Golden Fish (Carassius auratus) and Trail for its Treatment in Earthen Ponds at Kafr El-Sheikh Governorate, Egypt. Global Veterinaria 11(5):521-527.

Pavanelli G.C., Eiras J.C. \& Takemoto R.M. 2008. Doenças de Peixes: profilaxia, diagnóstico e tratamento. 3a ed. Eduem, Maringá. 331p.

Pavanelli G.C., Takemoto R.M. \& Eiras J.C. 2013. Parasitologia de Peixes de Água Doce do Brasil. Eduem, Maringá. 452p.

Rocha M.A., Ribeiro E.L.A., Mizubuti I.Y., Silva L.D.F., Borosky J.C. \& Rubin K.C.P. 2005. Uso do fator de condição alométrico e de fulton na comparação de carpa (Cyprinus carpio), considerando os sexos e idade. Semina: Ciênc. Agrárias 26(3):429-434.

Satake F., Pádua S.B. \& Ishikawa M.M. 2009. Distúrbios morfológicos em células sanguíneas de peixes em cultivo: uma ferramenta prognóstica, p.330-345. In: Tavares-Dias M. (Ed.), Manejo e Sanidade de Peixes em Cultivo. Embrapa Amapá, Macapá.

Tavares-Dias M., Ishikawa M.M., Martins M.L., Satake F., Hisano H., Pádua S.B., Jerônimo G.T. \& Sant'ana A.R. 2009. Hematologia: ferramenta para o monitoramento do estado de saúde de peixes em cultivo, p.43-80. In: Saran-Neto, Mariano \& Pozzobon-Soria (Eds), Tópicos Especiais em Saúde e Criação Animal. Pedro and João Editores, São Carlos.

Vasconcelos H.C.G. \& Tavares-Dias M. 2014. Influência da sazonalidade na infestação de Ergasilus turucuyus (Copepoda: Ergasilidae) em Acestrorhynchus falcirostris e Hemiodus unimaculatus (Osteichthyes: Characiformes) do Reservatório Coaracy Nunes, estado do Amapá, Brasil. Biota Amazônia 4(1):106-110.

Zanolo R. \& Yamamura M.H. 2006. Parasitas em Tilápias-do-Nilo criadas em sistema de tanques-rede. Semina: Ciênc. Agrárias 27(2):281-288. 